Open Access

\title{
Clinical impact of high-attenuation and cystic areas on computed tomography in fibrotic idiopathic interstitial pneumonias
}

Kiminobu Tanizawa' ${ }^{2}$, Tomohiro Handa ${ }^{2}$, Sonoko Nagai ${ }^{3}$, Toyohiro Hirai ${ }^{2,3^{*}}$, Takeshi Kubo ${ }^{4}$, Tsuyoshi Oguma ${ }^{2}$,

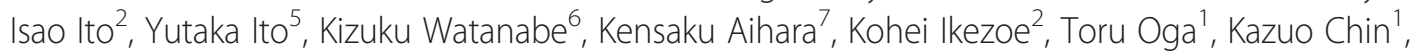

Takateru Izumi ${ }^{3}$ and Michiaki Mishima ${ }^{1}$

\begin{abstract}
Background: Quantitative computed tomography (CT) analysis has been proposed as a means of objectively assessing fibrotic interstitial pneumonia (IP) including idiopathic pulmonary fibrosis (IPF). We investigated whether percentages of high-attenuation areas (HAA\%) and cystic areas (CA\%) quantified from CT images were useful as indices of fibrotic IP.

Methods: CT images of 74 patients with fibrotic idiopathic interstitial pneumonias (IPF, 36; non-specific interstitial pneumonia, 9; unclassifiable idiopathic interstitial pneumonia, 29) were analyzed via in-house computer software, which automatically calculated HAA\%, CA\%, mean lung density (MLD), standard deviation of lung density (SD-LD), kurtosis, and skewness from CT attenuation histograms. These indices were compared in each instance with physiologic measures, visual fibrosis score, clinical diagnosis, radiologic CT pattern, and prognosis.

Results: HAA\% correlated significantly with physiologic measures and visual fibrosis score to a moderate extent (\%forced vital capacity, $r_{s}=-0.59$; \% carbon monoxide diffusion capacity, $r_{s}=-0.43$; fibrosis score, $r_{s}=0.23$ ). Densitometric parameters (MLD, SD-LD, kurtosis, and skewness) correlated significantly with physiologic measures and fibrosis score $\left(\left|r_{s}\right|=0.28-0.59\right)$. CA\% showed no association with pulmonary functions but differed significantly between IPF and other interstitial pneumonias (IPS) $(1.50 \pm 2.41 \%$ vs. $0.41 \pm 0.80 \% ; P<0.01)$ and between the definite usual interstitial pneumonia (UIP) pattern and other patterns (1.48 $\pm 2.38 \%$ vs. $0.55 \pm 1.19 \% ; P<0.01)$. On univariate analysis, HAA\%, MLD, SD-LD, kurtosis, skewness, fibrosis score, and definite UIP pattern all correlated with survival, with kurtosis alone identified as a significant predictor of mortality on multivariate analysis (hazard ratio $=0.67 ; 95 \% \mathrm{Cl}$, 0.44-0.96; $P=0.03$.
\end{abstract}

Conclusion: $C A \%$ and HAA\% are novel quantitative $C T$ indices with differing properties in fibrotic IP evaluations. HAA\% largely reflects physiologic impairments, whereas CA\% corresponds with diagnosis and HRCT pattern. Of the CT indices examined, kurtosis constituted the strongest predictor of mortality.

Keywords: Quantitative computed tomography, Densitometry, Idiopathic interstitial pneumonias, Prognosis

\footnotetext{
*Correspondence: t_hirai@kuhp.kyoto-u.ac.jp

${ }^{2}$ Department of Respiratory Medicine, Graduate School of Medicine, Kyoto University, 54 Shogoin Kawaharacho, Sakyo-ku, Kyoto 606-8507, Japan

${ }^{3}$ Kyoto Central Clinic, Clinical Research Center, Kyoto, Japan

Full list of author information is available at the end of the article
} 


\section{Background}

Quantitative high-resolution computed tomography (HRCT) analysis of the lung has been proposed as an objective and non-invasive means of assessing parenchymal lesions in fibrotic interstitial lung diseases (ILDs) [1-5]. Densitometric parameters (such as mean lung density [MLD], standard deviation of lung density [SD-LD], kurtosis, and skewness) derived from $\mathrm{CT}$ attenuation histograms have served as indices in earlier studies [1-6], corresponding with histopathologic diagnosis [3], physiologic impairment [1, 2, 4, 6], and healthrelated quality of life [4] in fibrotic ILDs, and with survival in idiopathic pulmonary fibrosis (IPF) [5]. However, no standard quantitative method for assessing fibrotic ILDs (IPF and others) by CT has been adopted as yet, nor has the clinical utility of this approach been firmly established [7].

Fibrotic ILDs are marked by a combination of radiologic abnormalities, with high and low CT attenuation [1]. Typically, high-attenuation areas (HAAs) signify parenchymal lesions, such as ground-glass opacity (GGO) and reticulation [8], whereas emphysematous change and cystic areas (CAs) are signified by low-attenuation areas (LAAs). CAs with honeycombing are more characteristic of fibrotic ILDs [8]. Determining percentages of HAAs (HAA\%) and CAs (CA\%) in whole lung fields may aid in determining the extents these characteristic lesions. It was our view that $\mathrm{HAA} \%$ and $\mathrm{CA} \%$ might serve as quantitative CT indices of fibrotic ILDs.

The main purpose of the present study was to determine the clinical utility of HAA\% and CA\% (alongside densitometric parameters) in assessing fibrotic interstitial pneumonia (IP). The relationships of these $\mathrm{CT}$ indices with physiologic impairment, visual score, clinical diagnosis, CT pattern, and prognosis were examined.

\section{Methods \\ Patients}

For this retrospective study, 74 consecutive patients with fibrotic idiopathic interstitial pneumonias (fibrotic IIPs) were recruited. All patients were undergoing HRCT at Kyoto Central Clinic, Kyoto, Japan between January 2004 and December 2006 and were followed for $>3$ months. Fibrotic IIPs comprised IPF, non-specific interstitial pneumonia (NSIP; biopsy-proven in all) and unclassifiable IIPs. IPF and NSIP were diagnosed according to the 2002 American Thoracic Society (ATS)/European Respiratory Society (ERS) IIP statement [9], and HRCT patterns were classified based on the 2011 American Thoracic Society (ATS)/European Respiratory Society (ERS)/Japanese Respiratory Society (JRS)/Latin American Thoracic Association (LATA) IPF guidelines [7]. If HRCT showed a possible or inconsistent usual interstitial pneumonia (UIP) pattern, and a pathologic diagnosis was unavailable, the case was interpreted as unclassifiable IIP according to the 2013 ATS/ERS IIP statement [10]. Patients were excluded on grounds of connective tissue disease or systemic vasculitis, history of exposure to any causative agent of ILD, active pulmonary infection, acute respiratory illness in the preceding 4 weeks, or viable neoplasm. The Kyoto Central Clinic Institutional Review Board approved this study protocol. Written informed consent was not obtained from the participants, because this is a retrospective study using clinical and HRCT data that were accumulated in daily practice.

\section{Clinical evaluation}

Clinical information was collected from medical records. Standardized pulmonary function tests and HRCT were performed on the same day [11]. Equations published for Japanese adults were used to determine the predicted values of each parameter [12].

\section{HRCT techniques}

In each instance, thin-section HRCT was done using a CT scanner with single-detector row (Pronto; Hitachi Medical Corporation, Tokyo, Japan) at $120 \mathrm{kVp}, 200 \mathrm{mAs}$, and 33$\mathrm{cm}$ field of view settings. Axial scans $(2 \mathrm{~mm}$ thick) were obtained at $10-\mathrm{mm}$ intervals, with a gantry speed of $1.0 \mathrm{~s} /$ rotation. No contrast medium was used. In the course of scanning, breath-holding was required after deep inspiration in supine position. Each HRCT image generated a $512 \times 512$ matrix of numeric data (CT numbers) in Hounsfield units (HU) via standard lung algorithm (filter No. 9). In addition to routine calibration by air and water phantoms, CT numbers were corrected using air density samples from intrathoracic trachea to eliminate effects of $\mathrm{X}$-ray tube aging [13].

\section{Quantitative CT analysis}

In-house computer software was engaged to analyze all HRCT lung images. Lung fields in each slice were identified by excluding major hilar bronchi and vessels. HAAs and LAAs were defined as areas in lung fields with CT values $>-200 \mathrm{HU}$ and $<-960 \mathrm{HU}$, respectively. LAAs were indicative of emphysematous patches, and in general CAs were equated with honeycombing. To extract CAs from LAAs, each LAA cluster was first defined as a continuous LAA entirely bounded by pixels, with CT values $>-960 \mathrm{HU}$. Most emphysematous lesions were small, discrete LAAs or larger LAA clusters, rather than cystic lesions. Cystic lesions only, particularly areas of honeycombing, were defined as LAA clusters with areas of $9 \pi-400 \pi \mathrm{mm}^{2}$ (i.e., circular areas $3-20 \mathrm{~mm}$ in diameter) (Fig. 1). The minimum diameter $(3 \mathrm{~mm})$ was stipulated by a recent radiologic definition of honeycombing [14], and the maximum diameter $(20 \mathrm{~mm})$ was set to exclude continuous emphysematous lesions and bullous changes. HAA\% and CA\% were calculated as percentages of whole lung field occupied by HAA and CA, respectively. MLD, 


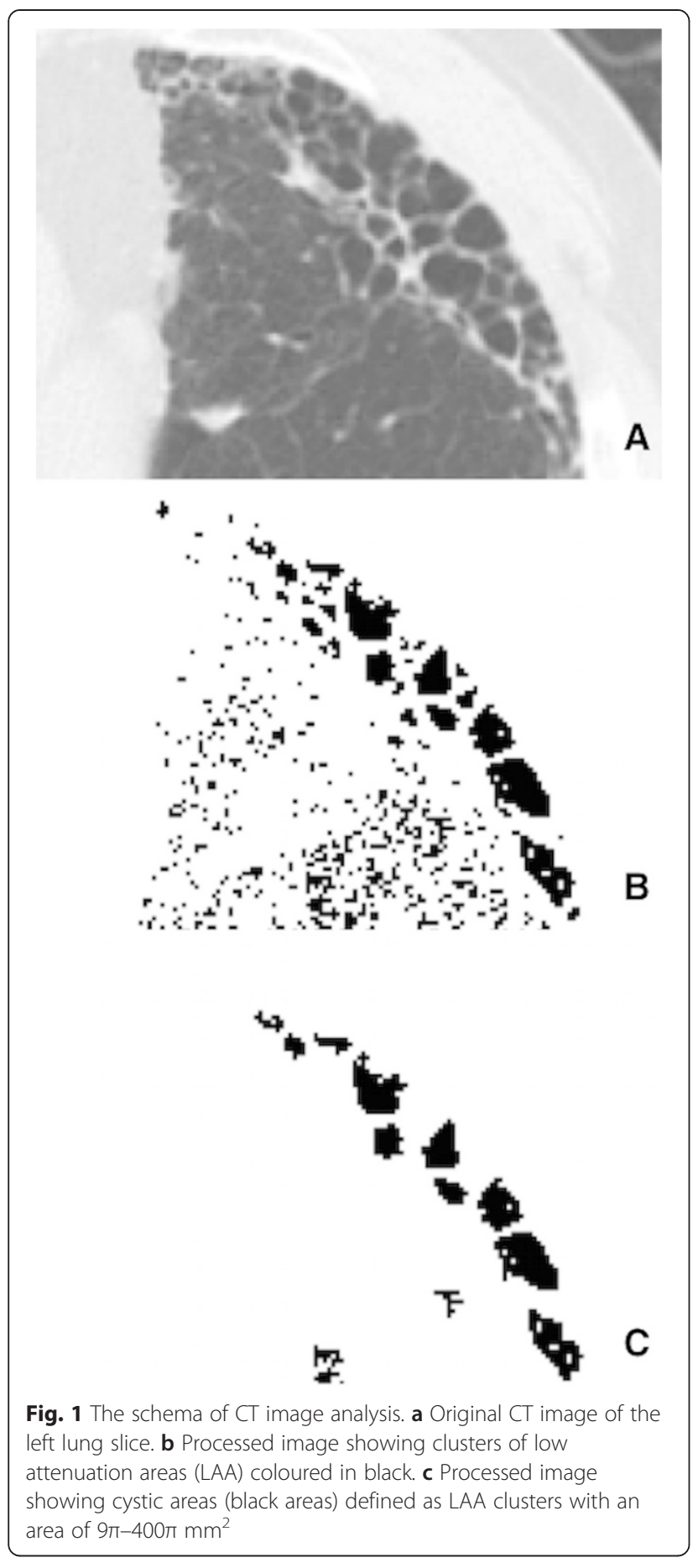

SD-LD, kurtosis, and skewness likewise were calculated automatically from CT attenuation histograms as follows:

$$
\operatorname{MLD}=\sum_{l=l \min }^{l \max } \ln (l) / N
$$

$$
\begin{aligned}
& \text { SD-LD }=\sqrt{\sum_{l=l \min }^{l_{\text {max }}} n(l)(l-M L D)^{2} / N} \\
& \text { Kurtosis }=\sum_{l=l \text { min }}^{l \max }\left\{n(l)(l-M L D)^{4} / N(S D)^{4}\right\}-3 \\
& \text { Skewness }=\sum_{l=l_{\min }}^{l_{\max }}\left\{n(l)(l-M L D)^{3} / N(S D)^{3}\right\}
\end{aligned}
$$

\section{$1=\mathrm{CT}$ value}

$\mathrm{n}(l)=$ number of pixels in each $C T$ value

$\mathrm{N}=$ number of pixels in all CT values

$\mathrm{SD}=\mathrm{SD}-\mathrm{LD}$

MLD and SD-LD represent the average and standard deviation of the HU of each pixel, respectively. Kurtosis describes how sharply peaked a histogram is when compared with the histogram of a normal distribution. Skewness describes the degree of asymmetry of a histogram, and a long right tail indicates positive skewness.

\section{CT visual scoring}

Three independent observers (KT, TH, and TK) blinded to clinical information reviewed HRCT images. Lung fields were divided into upper, middle, and lower zones at level of carina and at right inferior pulmonary venous confluence, respectively. On a scale of $0-5$, each of the three zones was rated for extent of GGO (GGO score) and fibrotic opacity (fibrosis score) [15]. Mean scores for each zone and for the whole lung were calculated jointly by the three observers.

\section{Statistical analyses}

Statistical analyses relied on standard software (JMP v9; SAS Institute Inc., Cary, NC, USA). Each statistical variation in quantitative data was expressed as a single determination \pm standard deviation, with statistical significance set at $P<0.05$. Spearman's rank correlation test was used to examine the relationships between quantitative CT indices, physiologic measures, and CT visual scores. Group comparisons were made using the Mann-Whitney $U$ test. Univariate and multivariate survival regression analyses were performed to assess prognostic value of each CT index, applying the Cox proportional hazards model.

\section{Results}

\section{Characteristics of study patients}

Characteristics of study population are summarized in Table 1. Among the 75 patients with fibrotic IIPs, the diagnoses were IPF $(n=36,12$ biopsy proven), nonspecific interstitial pneumonia (NSIP; $n=9$, all biopsy proven), and unclassifiable IIP $(n=29)$. On HRCT, definite UIP pattern was observed in 28 (78 \%) of IPF group 
Table 1 Characteristics of study population $(n=74)$

\begin{tabular}{|c|c|c|}
\hline Characteristics & Value & Range \\
\hline \multicolumn{3}{|l|}{ Demographics } \\
\hline Age, years & $65.7 \pm 9.2$ & $(30-86)$ \\
\hline Male & 49 (66 \%) & - \\
\hline Smoking (yes) & $45(60 \%)$ & \\
\hline Interval from diagnosis, months & $58.1 \pm 48.2$ & $(1-263)$ \\
\hline \multicolumn{3}{|l|}{ Diagnosis } \\
\hline IPF & $36(49 \%)$ & \\
\hline NSIP & $9(12 \%)$ & \\
\hline Other & $29(39 \%)$ & \\
\hline \multicolumn{3}{|l|}{ Pulmonary function tests } \\
\hline \%FVC, \% & $72.2 \pm 20.0$ & $(29.0-113.3)$ \\
\hline$\% \mathrm{DLCO}, \%$ & $54.1 \pm 21.8$ & $(10.3-122.9)$ \\
\hline \multicolumn{3}{|l|}{ Quantitative $C T$ indices } \\
\hline HAA\% (>-200 HU), \% & $1.83 \pm 1.15$ & $(0.27-7.01)$ \\
\hline LAA\% (<-960 HU), \% & $3.91 \pm 4.25$ & $(0.14-22.5)$ \\
\hline CA\%, \% & $0.94 \pm 1.85$ & $(0-10.54)$ \\
\hline MLD, HU & $-758.0 \pm 53.3$ & $(-860.3--635.7)$ \\
\hline SD-LD, HU & $195.9 \pm 22.6$ & $(152.5-259.4)$ \\
\hline Kurtosis & $2.40 \pm 1.81$ & $(-0.53-7.45)$ \\
\hline Skewness & $1.61 \pm 0.44$ & $(0.66-2.68)$ \\
\hline \multicolumn{3}{|l|}{$C T$ visual scores } \\
\hline GGO score $[0-5]$ & $0.45 \pm 0.52$ & $(0-2.06)$ \\
\hline Fibrosis score [0-5] & $1.66 \pm 0.68$ & $(0.33-3.28)$ \\
\hline Fibrosis score $\geq 2$ in any field & $48(65 \%)$ & \\
\hline
\end{tabular}

Values expressed as mean \pm standard deviation or number (\%) Numbers in square brackets represent theoretical score range IPF idiopathic pulmonary fibrosis, NSIP, nonspecific interstitial pneumonia; $\% F V C$ percentage of predicted forced vital capacity, \%DLCO percentage of predicted diffusion capacity of carbon monoxide, CT computed tomography, $H A A \%$ percentage of lung field occupied by high-attenuation areas, LAA\% percentage of lung field occupied by low-attenuation areas, $C A \%$ percentage of lung field occupied by cystic areas, MLD mean lung density, HU hounsfield units, $S D-L D$ standard deviation of lung densities, $G G O$ ground-glass opacity

members; and of 29 subjects with unclassifiable IIP, 8 (28\%) displayed possible UIP pattern and 21 (72 \%) inconsistent UIP. Mean interval from diagnosis to HRCT evaluation was 58.1 months (range, 5-86 months).

\section{Comparisons of quantitative $\mathrm{CT}$ indices, physiologic measures, and $\mathrm{CT}$ visual scores}

The relationships of quantitative $\mathrm{CT}$ indices with physiologic impairment and semi-quantitative visual assessment of CT images are presented in Table 2. The interobserver correlation coefficients of the GGO and fibrosis scores calculated by the Blant-Altoman method were $0.72-0.83$ and $0.77-0.95$, respectively. The interobserver Spearman's rank correlation coefficients $\left(\mathrm{r}_{\mathrm{s}}\right)$ of the GGO and fibrosis scores were $0.66-0.77$ and $0.77-0.96$, respectively. HAA\% correlated moderately with physiologic
Table 2 Spearman's rank correlation coefficients for determinants of fibrotic IP

\begin{tabular}{lcccccc}
\hline & HAA\% & CA\% & MLD & SD-LD & Kurtosis & Skewness \\
\%FVC & -0.59 & - & -0.55 & -0.52 & 0.48 & 0.49 \\
\hline \%DLCO & -0.43 & - & -0.56 & -0.45 & 0.57 & 0.59 \\
GGO & - & -0.40 & - & - & - & - \\
Fibrosis & 0.25 & 0.38 & - & 0.33 & -0.28 & -0.28
\end{tabular}

Missing data (-) indicating correlation was not statistically significant $C T$ computed tomography, HAA\% percentage of lung field occupied by highattenuation areas, $C A \%$ percentage of lung field occupied by cystic areas, MLD mean lung density, $H U$ hounsfield units, $S D-L D$ standard deviation of lung densities, \%FVC percentage of predicted forced vital capacity, \%DLCO percentage of predicted diffusion capacity of carbon monoxide, GGO visual score of ground-glass opacity, Fibrosis, visual fibrosis score

measures $\left(\left|\mathrm{r}_{\mathrm{s}}\right|=0.43-0.59\right)$ and weakly with fibrosis score $\left(\left|r_{s}\right|=0.25\right)$. Densitometric parameters correlated moderately with both physiologic measures $\left(\left|\mathrm{r}_{\mathrm{s}}\right|=0.45-0.59\right)$ and fibrosis score $\left(\left|r_{s}\right|=0.28-0.33\right)$, whereas CA\% correlated weakly with fibrosis score $\left(r_{s}=0.35\right)$ and held no relationship with physiologic impairment.

\section{Comparison of quantitative CT indices in IPF and non-IPF groups}

To determine whether quantitative CT indices reflected differences in clinical diagnosis, IPF $(n=36)$ and non-IPF $\quad(n$ $=38$, i.e., NSIP and unclassifiable IIPs) group values were compared (Table 3). CA\% of the IPF group significantly exceeded that of the non-IPF group $(1.50 \pm 2.41 \%$ vs. $0.41 \pm 0.80 \% ; P<0.01)$, but HAA\% and densitometric parameters of IPF and non-IPF groups were similar.

Quantitative CT indices of subjects with definite UIP pattern $(n=35)$ and those with other patterns $(n=39)$ on HRCT (Table 4) were also compared. Again, CA\% was significantly higher in patients with definite UIP pattern $(1.48 \pm 2.38 \%$ vs. $0.55 \pm 1.19 \% ; P<0.01)$, whereas HAA\% and densitometric parameters did not differ between groups.

\section{Prognostic value of quantitative $\mathrm{CT}$ analysis}

Median duration of follow-up after HRCT was 38 months (range, 5-86 months), and survivors were followed up for 8-86 months (median, 66 months). Of subjects studied, 31 (41 \%) died (chronic respiratory failure, 13; acute exacerbation, 12; pulmonary infections, 4; pneumothorax, 1 ; sudden death, 1).

Univariate regression analyses indicated that HAA\%, MLD, SD-MLD, kurtosis, skewness, fibrosis score, and definite UIP pattern corresponded significantly with poorer prognosis (Table 5), whereas CA\% was not a significant predictor of mortality. Given these results, HAA\%, kurtosis, fibrosis score, and definite UIP pattern were entered into the Cox proportional hazards model. Kurtosis was the sole densitometric parameter entered, because it correlated strongly with other parameters 
Table 3 Comparison of subject groups: IPF vs non-IPF

\begin{tabular}{llll}
\hline & $\operatorname{IPF}(n=36)$ & Non-IPF $(n=38)$ & $P$ \\
\hline Pulmonary function tests & & & \\
\%FVC, \% & $71.5 \pm 20.7$ & $72.9 \pm 19.5$ & NS \\
\%DLCO, \% & $56.0 \pm 23.9$ & $52.5 \pm 19.9$ & NS \\
Quantitative CT indices & & & \\
HAA\% (>-200 HU), \% & $1.90 \pm 1.31$ & $1.76 \pm 0.99$ & NS \\
LAA\% (<-960 HU), \% & $5.25 \pm 5.17$ & $2.64 \pm 2.62$ & $<0.01$ \\
CA\%, \% & $1.50 \pm 2.41$ & $0.41 \pm 0.80$ & $<0.01$ \\
MLD, HU & $-767.4 \pm 51.0$ & $-749.1 \pm 54.6$ & NS \\
SD-LD, HU & $197.6 \pm 24.6$ & $194.3 \pm 20.7$ & NS \\
Kurtosis & $2.54 \pm 1.87$ & $2.21 \pm 1.82$ & NS \\
Skewness & $1.67 \pm 0.44$ & $1.56 \pm 0.45$ & NS \\
CT visual scores & & & \\
GGO score [0-5] & $0.21 \pm 0.25$ & $0.67 \pm 0.61$ & $<0.01$ \\
Fibrosis score [0-5] & $2.10 \pm 0.44$ & $1.23 \pm 0.59$ & $<0.01$ \\
Fibrosis score $\geq 2$ in any field & $35(97 \%)$ & $13(34 \%)$ & $<0.01$ \\
\hline
\end{tabular}

Values expressed as mean \pm standard deviation or number (\%) Numbers in square brackets represent theoretical score range IPF idiopathic pulmonary fibrosis, NSIP nonspecific interstitial pneumonia, $\% F V C$ percentage of predicted forced vital capacity, \%DLCO percentage of predicted diffusion capacity of carbon monoxide, $C T$ computed tomography, $H A A \%$ percentage of lung field occupied by high-attenuation areas, LAA\% percentage of lung field occupied by low-attenuation areas, $C A \%$ percentage of lung field occupied by cystic areas, MLD mean lung density, HU hounsfield units, $S D-L D$ standard deviation of lung densities, GGO ground-glass opacity $\left(\left|r_{s}\right|=0.85-0.98\right)$, and another study in IPF recognized its superiority in predicting mortality [5]. In multivariate analysis, kurtosis was the only significant determinant of prognosis $\quad(\mathrm{HR}=0.67 ; 95 \% \mathrm{CI}, \quad 0.44-0.96 ; \quad P=0.03)$ (Table 5).

To assess the independent prognostic value of kurtosis, multivariate regression analyses were performed, coupling kurtosis with a second clinical parameter (Table 6). Kurtosis remained a significant prognostic factor, even after adjusting for age, male gender, and clinical diagnosis of IPF separately (Table 6, models 1-3). Kurtosis was also a stronger predictor of mortality than \%FVC (Table 6, model 4), albeit surpassed by \%DLCO.

\section{Discussion}

Through this study, we found that HAA\% and densitometric parameters were associated with physiologic measures and with CT visual scores in fibrotic IP, whereas CA\% helped to distinguish between IPF and non-IPF diagnoses. High HAA\% was also associated with survival, but among CT indices, kurtosis was the most significant predictor of mortality. These outcomes validate use of HAA\% and $\mathrm{CA} \%$ as indices with which to quantify parenchymal lesions of fibrotic IP in CT images.

A novel CT index, HAA\%, was introduced herein to gauge the extent of parenchymal abnormalities (primarily fibrotic lesions) in fibrotic IP [8]. Restrictive pulmonary function (reduction in \%FVC) and impaired gas exchange (diminished \%DLCO) are the major physiologic

Table 4 Comparison of subject groups: definite UIP (HRCT) vs other patterns

\begin{tabular}{|c|c|c|c|}
\hline & Definite UIP $(n=35)$ & Other patterns $(n=39)$ & $P$ \\
\hline \multicolumn{4}{|l|}{ Pulmonary function tests } \\
\hline$\% F V C, \%$ & $71.7 \pm 20.6$ & $73.0 \pm 19.4$ & NS \\
\hline$\% \mathrm{DLCO}, \%$ & $54.8 \pm 23.4$ & $53.5 \pm 20.6$ & NS \\
\hline \multicolumn{4}{|l|}{ Quantitative $C T$ indices } \\
\hline HAA\% (>-200 HU), \% & $1.93 \pm 1.32$ & $1.74 \pm 0.98$ & NS \\
\hline LAA\% (<-960 HU), \% & $5.34 \pm 5.22$ & $2.63 \pm 2.59$ & $<0.01$ \\
\hline CA\%, \% & $1.54 \pm 2.43$ & $0.40 \pm 0.79$ & $<0.01$ \\
\hline MLD, HU & $-765.8 \pm 50.9$ & $-751.0 \pm 55.2$ & NS \\
\hline SD-LD, HU & $198.2 \pm 24.8$ & $193.9 \pm 20.6$ & NS \\
\hline Kurtosis & $2.50 \pm 1.81$ & $2.25 \pm 1.82$ & NS \\
\hline Skewness & $1.66 \pm 0.44$ & $1.58 \pm 0.45$ & NS \\
\hline \multicolumn{4}{|l|}{ CT visual scores } \\
\hline GGO score [0-5] & $0.21 \pm 0.25$ & $0.66 \pm 0.61$ & $<0.01$ \\
\hline Fibrosis score [0-5] & $2.13 \pm 0.41$ & $1.23 \pm 0.50$ & $<0.01$ \\
\hline Fibrosis score $\geq 2$ in any field & $35(100 \%)$ & $13(33 \%)$ & $<0.01$ \\
\hline
\end{tabular}

Values expressed as mean \pm standard deviation or number (\%)

Numbers in square brackets represent theoretical score range

IPF idiopathic pulmonary fibrosis, NSIP nonspecific interstitial pneumonia, \%FVC percentage of predicted forced vital capacity, \%DLCO percentage of predicted diffusion capacity of carbon monoxide, $C T$ computed tomography, $H A A \%$ percentage of lung field occupied by high-attenuation areas, $L A A \%$ percentage of lung field occupied by low-attenuation areas, $C A \%$ percentage of lung field occupied by cystic areas, MLD mean lung density, $H U$ hounsfield units, $S D$ - $L D$ standard deviation of lung densities, GGO ground-glass opacity 
Table 5 Univariate and multivariate regression analyses: radiologic indices of survival $(n=74)$

\begin{tabular}{|c|c|c|c|c|c|c|}
\hline & \multicolumn{3}{|c|}{ Univariate } & \multicolumn{3}{|c|}{ Multivariate } \\
\hline & $\mathrm{HR}$ & $95 \% \mathrm{Cl}$ & $P$ & $\mathrm{HR}$ & $95 \% \mathrm{Cl}$ & $P$ \\
\hline \multicolumn{7}{|l|}{ Quantitative $\mathrm{CT}$ indices } \\
\hline HAA\% (>-200 HU), \% & 1.47 & $(1.13-1.83)$ & $<0.01$ & 1.06 & $(0.86-1.06)$ & NS \\
\hline LAA\% (<-960 HU), \% & 0.97 & $(0.86-1.06)$ & NS & & & \\
\hline CA\%, \% & 0.98 & $(0.75-1.16)$ & NS & & & \\
\hline MLD, HU & 1.01 & $(1.00-1.02)$ & 0.01 & & & \\
\hline SD-LD, HU & 1.03 & $(1.01-1.04)$ & $<0.01$ & & & \\
\hline Kurtosis & 0.67 & $(0.51-0.86)$ & $<0.01$ & 0.67 & $(0.44-0.96)$ & 0.03 \\
\hline Skewness & 0.28 & $(0.11-0.65)$ & $<0.01$ & & & \\
\hline \multicolumn{7}{|l|}{$C T$ visual scores } \\
\hline GGO score & 0.74 & $(0.35-1.40)$ & NS & & & \\
\hline Fibrosis score & 2.00 & $(1.20-3.39)$ & 0.01 & 1.06 & $(0.45-2.21)$ & NS \\
\hline Definite UIP pattern & 2.13 & $(1.05-4.47)$ & 0.04 & 2.36 & $(0.84-7.60)$ & NS \\
\hline
\end{tabular}

$H R$ hazard ratio, $C l$ confidence interval, $C T$ computed tomography, $H A A \%$ percentage of lung field occupied by high-attenuation areas, $C A \%$ percentage of lung field occupied by cystic areas, MLD mean lung density, HU hounsfield units, SD-LD standard deviation of lung densities, GGO ground-glass opacity, UIP usual interstitial pneumonia

Table 6 Multivariate survival regression analyses: kurtosis and other clinical parameters

\begin{tabular}{|c|c|c|c|}
\hline \multicolumn{4}{|l|}{ Model 1} \\
\hline & $\mathrm{HR}$ & $95 \% \mathrm{Cl}$ & P \\
\hline Age & 1.01 & $(0.97-1.06)$ & 0.54 \\
\hline Kurtosis & 0.67 & $(0.51-0.86)$ & $<0.01$ \\
\hline \multicolumn{4}{|l|}{ Model 2} \\
\hline & $\mathrm{HR}$ & $95 \% \mathrm{Cl}$ & P \\
\hline Male & 2.96 & $(1.23-8.78)$ & 0.01 \\
\hline Kurtosis & 0.66 & $(0.50-0.85)$ & $<0.01$ \\
\hline \multicolumn{4}{|l|}{ Model 3} \\
\hline & $\mathrm{HR}$ & $95 \% \mathrm{Cl}$ & P \\
\hline IPF & 2.85 & $(1.37-6.17)$ & $<0.01$ \\
\hline Kurtosis & 0.62 & $(0.46-0.81)$ & $<0.01$ \\
\hline \multicolumn{4}{|l|}{ Model 4} \\
\hline & $\mathrm{HR}$ & $95 \% \mathrm{Cl}$ & P \\
\hline$\% F V C$ & 0.98 & $(0.96-1.00)$ & 0.05 \\
\hline Kurtosis & 0.75 & $(0.55-0.97)$ & 0.03 \\
\hline \multicolumn{4}{|l|}{ Model 5} \\
\hline & $\mathrm{HR}$ & $95 \% \mathrm{Cl}$ & P \\
\hline \%DLCO & 0.96 & $(0.93-0.99)$ & 0.01 \\
\hline Kurtosis & 0.81 & $(0.57-1.07)$ & 0.15 \\
\hline
\end{tabular}

HR hazard ratio, $\mathrm{Cl}$ confidence interval, IPF idiopathic pulmonary fibrosis, \%FVC percentage of predicted forced vital capacity, $\% D L C O$ percentage of predicted diffusion capacity of carbon monoxide impairments in fibrotic IP; as anticipated, HAA\% corresponded with both, similar to conventional densitometric parameters. Correlations between densitometric parameters and physiologic indices have been reported in IPF $[1,2]$, in asbestosis [1], and in scleroderma [4]. Our data generated from CT histograms have extended these findings to a more heterogeneous group of fibrotic IIPs, adding HAA\% to current battery of available parameters. Because HAA\% reflects the extent of parenchymal lesions, such as GGO and reticulation [8], it appears that quantifying the degree of fibrotic changes determines the physiologic burden of fibrotic IP. This concept is aligned with a previous study in IPF where semi-quantitative scoring of fibrotic lesions was done [16]. The impact of HAA\% and densitometric parameters on survival, shown in univariate analysis, also validates HAA\% as a clinically relevant CT determinant of fibrotic IP.

The second new CT index introduced, largely coinciding with extent of honeycombing, was CA\%. Unlike $\mathrm{HAA} \%$ and densitometric parameters, CA\% seemed to reflect clinical diagnostic and radiologic pattern differences and thus may be useful for distinguishing IPF and definite UIP pattern from other entities. The significant correlation shown between CA\% and fibrosis score (i.e., visually scored honeycombing) suggests that CA\% may capture and quantify characteristic lesions of fibrotic IP. On the other hand, we found no association between CA\% and \%FVC or \%DLCO. Although restrictive impairment and extent of honeycombing did not correlate in other studies of IPF $[16,17]$, significant correlations between honeycombing and impaired gas exchange were consistently identified, using semi-quantitative scoring [16] and a new quantitative CT method, texture analysis 
[17]. In terms of survival, our outcomes also differed from these studies, which showed that degree of honeycombing significantly predicted mortality in IPF. There was no relationship between CA\% and survival in our cohort. These discrepancies raise the possibility that our method of determining CA\% differed somewhat from assessing honeycombing visually, although CA\% correlated significantly with fibrosis score in our study. Further studies are needed to define the properties of CA\% and to refine the measuring of cystic lesions and honeycombing in fibrotic IP.

Among quantitative CT indices, only MLD failed to correlate with fibrosis score. As honeycombing or LAA expands, fibrosis score increases, whereas MLD may remain unchanged or decrease. The reason is that increments in LAA (including honeycombing) offset any fluctuations in HAA. Although MLD is associated with physiologic measures and survival, this dynamic suggests a possible flaw in using MLD as an integrative index of fibrotic and cystic lesions in fibrotic IP. MLD properties differing from those other densitometric parameters were also reported in distinguishing IPF from NSIP [3].

Our analysis of CT parameters identified kurtosis as the strongest predictor of mortality in fibrotic IP. Univariate analysis also underscored that $\mathrm{HAA} \%$, fibrosis score, and definite UIP pattern were significant correlates of survival. These findings approximated those of another study of IPF, showing that kurtosis surpassed other densitometric parameters in this regard, although fibrosis score was the only variable of significance in multivariate analysis [5]. Consequently, it appears that quantitative CT indices, including HAA\%, enable assessment of disease burden in fibrotic IP and may signal long-term outcomes, making them potential surrogate markers for clinical trials. In addition, kurtosis was independent of other clinical and physiologic parameters in its prognostic capacity, except for \%DLCO. Given the difficulty in measuring \%DLCO in patients with severe respiratory failure, these $\mathrm{CT}$ indices may more readily serve analogous roles in clinical trials and other settings.

The pathological background of these CT indices has been investigated in prior studies. Do et al. reported that kurtosis and skewness were higher in patients with pathological UIP than in those with NSIP [3]. Sumikawa et al. revealed that the histograms of GGA and fine reticulation patterns were similar, while the honeycombing pattern showed less kurtosis and skewness and a higher contrast and variance [18]. On the other hand, they also showed that the histogram of the whole lung was similar between UIP and NSIP, although an analysis of cubic regions of interest (ROIs) demonstrated differences between UIP and NSIP [19]. Those findings suggest that the whole lungs of patients with ILD are combinations of various ILD-characteristic regions. Although the histogram of each region can reflect the differences among the regions, the features of different ROIs offset each other in the histogram analysis of the whole lung, leading to conflicting results of comparisons between different pathological patterns $[3,20]$. Indeed, our results showed no significant differences in densitometric parameters between the IPF and non-IPF groups. Given the significant association between densitometric parameters and physiological impairments and long-term outcomes, the densitometric parameters of the whole lung might represent the physiological burdens of disease rather than pathological patterns. The novel CT indices used in this study, \%HAA and \%CA, are presumed to reflect the fibrotic and honeycombing lesions, respectively. The \%HAA was similar between the IPF and non-IPF groups. Although a different definition was used, the percentages of low, intermediate, and high CT density areas did not differ between UIP and NSIP in a previous study [20]. Similar to the densitometric parameters, the \%HAA or high-density area might not be an index for morphological characteristics but might instead be an index for the extent and severity of disease. Of note, the \%CA was higher in patients with IPF and correlated with the extent of honeycombing by visual scoring in our study. Those results suggest the possibility that the \%CA can detect the pathological features of IPF/UIP even in whole-lung analyses.

Quantitative CT analysis of the lung has been performed for COPD, bronchial asthma, and ILDs [6, 13, 21-26]. Histogram analysis of fibrotic ILDs has been conducted for IPF, asbestosis, and scleroderma [1, 2, 4, 5]; and MLD, SD-LD, kurtosis, and skewness were employed as CT indices in those studies. Sumikawa et al. added contrast, variance, and entropy to the repertory of CT indices used to discriminate the different ILDcharacteristic abnormalities more precisely [20]. In contrast to those for COPD and bronchial asthma, the standard CT indices remained to be elucidated for ILDs. In addition to the histogram indices, we calculated the $\% \mathrm{HAA}$ and \%CA. As aforementioned, the analysis of whole-lung histograms might not be able to detect the extents of different disease-characteristic lesions sufficiently, because each lesion can offset other lesions in a single histogram. Therefore, we sought to measure the areas of fibrotic lesions and honeycombing directly and automatically. Although such a cut-off approach using $\mathrm{CT}$ values has been well established in COPD and emphysema, its utility and limitations in ILDs should be examined in further studies.

Recently, texture analysis has emerged as a novel method for quantifying fibrotic IP by CT [17, 27]. Texture analysis is based on the histogram analysis of ILDcharacteristic findings in small ROIs. That method segments the whole lung into small ROIs, classifies each ROI into one CT pattern such as GGA, reticulation, or honeycombing determined through histogram analysis, 
and calculates the extent of each CT pattern automatically. As a result, the CT data of the whole lung are converted into a combination of the ROI percentages of the histogram-based CT patterns. Texture analysis aims to overcome the limitations of whole-lung histogram analysis by dividing the whole lung into small ROIs, thus avoiding the summation of the whole lung CT data into a single histogram. The expanse of honeycombed areas and serial changes in abnormalities (reticular and total interstitial) in texture analysis reportedly are significant predictors of mortality in IPF $[17,27]$. By comparison, percentages of reticular and honeycombed areas in whole lung fields of texture analysis exceeded corresponding \% HAA and \%CA values in our study, although the study groups were not comparable $[17,27]$. These texture analysis parameters also correlated more strongly with visual scores than did \%HAA and \%CA [17, 27]. Thus, ILD-specific lesions may be better defined via texture analysis than through our pixel-counting and global histogram approach.

On the other hand, texture analysis is a computeraided method, relying on recognition of radiologic features by a consensus of experts in each study group; indeed, software applications of the various studies were not uniform [17, 27]. Even among experts, inter-observer agreement on ILD-specific abnormalities, such as honeycombing, is less than satisfactory [28]. Thus, a gold standard of analytics has yet to be established, and the broader utility of texture analysis should be examined not only in IPF but also in other IIPs or fibrotic IP.

Another issue in quantitative CT analysis is the selection of ROIs. Sumikawa et al. reported that threedimensional (3D) histogram analysis using cubic ROIs is superior to two-dimensional histogram analysis with square ROIs for assessing various CT patterns of ILDs [18]. They applied a similar method to quantify pulmonary adenocarcinoma with GGA and demonstrated the utility of 3D histogram analysis in small lung cancer [29]. In addition, a 3D approach was used in a recent texture analysis [27]. We used CT scans with a $2 \mathrm{~mm}$ thickness obtained at $10 \mathrm{~mm}$ intervals and therefore could not apply a 3D analysis. Those differences in CT scanning conditions might have influenced our results.

There are acknowledged limitations to this study. First, the subjects did not receive uniform treatment such as corticosteroids, immunosuppressive agents, or pirfenidone, because evidence-based guidelines for IPF were just published recently [7]. Given the relatively poor outcomes of the patients with IPF who received the combination therapy of prednisone and azathioprine in the PANTHER-IPF study [30], different therapeutic strategies might have affected the long-term outcomes of patients with IPF. In addition, our cohort included several patients with non-IPF IIPs, whose responses to treatment could be more variable. Hence, we could not address the impact of therapeutic regimens on survival. Furthermore, the ramifications of dyspnea, overall health status, exercise capacity, and comorbidities could not be assessed, due to the retrospective design. These factors may well have bearing on prognosis of IPF and fibrotic IIPs [31-37]. Additionally, our cohort included several patients with unclassifiable IIP because of a lack of pathological diagnoses. Those patients can be potentially diagnosed with IPF or NSIP, and such diagnoses might influence the results of comparisons between IPF and non-IPF and multivariate survival analyses. Finally, we did not determine longitudinal changes in CT indices at this time. Best et al. have already demonstrated that serial changes in densitometric parameters correlate with changes in physiologic measures [5]. Given the significant impact of declining \% FVC on survival of patients with IPF [7, 36, 38, 39], the prognostic implications of changes in quantitative CT indices, particularly HAA\%, should be examined in future studies.

\section{Conclusion}

Despite these limitations, the differing properties of these novel quantitative CT indices, HAA\% and CA\%, were evident. HAA\% largely reflected physiologic impairments in fibrotic IP, whereas CA\% corresponded with diagnosis and HRCT pattern. Future studies applying quantitative CT analysis for fibrotic IP should incorporate these $\mathrm{CT}$ indices to assess disease characteristics and severity more comprehensively.

\section{Abbreviations}

\%DLCO: Percentage of predicted diffusion capacity of carbon monoxide; \%FVC: Percentage of predicted forced vital capacity; CA\%: Percentages of cystic areas; CAs: Cystic areas; Cl: Confidence interval; CT: Computed tomography; GGO: Ground-glass opacity; HAA\%: Percentages of high-attenuation areas; HAAs: High-attenuation areas; HR: Hazard ratio; HRCT: High-resolution computed tomography; HU: Hounsfield units; IIP: Idiopathic interstitial pneumonia; ILDs: Interstitial lung diseases; IP: Interstitial pneumonia; IPF: Idiopathic pulmonary fibrosis; LAA\%: Percentage of lung field occupied by low-attenuation areas; LAAs: Low-attenuation areas; MLD: Mean lung density; NSIP: Non-specific interstitial pneumonia; $r_{\text {s: }}$ Spearman's rank correlation coefficient; SD-LD: Standard deviation of lung density; UIP: Usual interstitial pneumonia.

\section{Competing interests}

The Department of Respiratory Care and Sleep Control Medicine at Kyoto University is funded by endowments from Philips Respironics, Teijin Pharma Ltd, Fukuda Denshi Inc. and Fukuda Lifetec Keiji. The authors themselves have no conflicts of interest to disclose.

\section{Authors' contributions}

KT collected, analyzed, and interpreted the data, and drafted the article. TH was fully responsible for data integrity and accuracy of data analysis, developing the concept and design of the study, collecting, analyzing, and interpreting the data, and critically revising the draft article. SN collected, analyzed, and interpreted data, revised the draft article critically, and gave final approval for the revised article. TH developed the in-house computer software, analyzed and interpreted data, and revised the draft article critically. KT collected, analyzed, and interpreted data, and revised the draft article critically. TO and II revised the draft article critically. YI collected, analyzed, and interpreted data and critically revised the draft article. KW, KA, Kl, and TO critically revised the draft article. $\mathrm{KC}, \mathrm{MI}$, and $\mathrm{MM}$ also revised the draft article, approving final revisions. 


\section{Acknowledgements}

Our thanks to Mr. M Saito, Mr. H Minami, Mr. K Sanbongi, Ms. N Matsuzaki, Mr. S Ueda, and Ms. M Hiramatsu for technical assistance; Ms. Y Sato, Ms. M Yokota, and Ms. S Yoshida for help in the outpatient clinic; and Ms. H Inoue, Ms. Y Kubo, Ms. I Morioka, Ms. S Shima, and Ms. N Chaki for secretarial help. We also thank Ms. T Toki, Ms. M Sotoda, Ms. N Kimura, and Ms. S Tamura for help with manuscript preparation.

\section{Funding}

This study was supported in part by a grant from Japan's Ministry of Health, Labor, and Welfare to the Diffuse Lung Diseases Research Group, the Respiratory Failure Study Group, and the Research Program of Intractable Disease. Other support included a KAKENHI Grant-in-Aid for Young Scientists (B), awarded by the Japan Society for the Promotion of Science, and grants from the Japan Intractable Diseases Research Foundation.

\section{Author details}

'Department of Respiratory Care and Sleep Medicine, Graduate School of Medicine, Kyoto University, Kyoto, Japan. ${ }^{2}$ Department of Respiratory Medicine, Graduate School of Medicine, Kyoto University, 54 Shogoin Kawaharacho, Sakyo-ku, Kyoto 606-8507, Japan. ${ }^{3}$ Kyoto Central Clinic, Clinical Research Center, Kyoto, Japan. ${ }^{4}$ Department of Diagnostic Imaging and Nuclear Medicine, Graduate School of Medicine, Kyoto University, Kyoto, Japan. ${ }^{5}$ Department of Respiratory Medicine, Allergy and Clinical Immunology, School of Medical Sciences, Nagoya City University, Nagoya, Japan. ${ }^{6}$ Department of Respiratory Medicine, Fukui Red-Cross Hospital, Fukui, Japan. ${ }^{7}$ Department of Respiratory Medicine, Saiseikai-Noe Hospital, Osaka, Japan.

Received: 16 February 2015 Accepted: 6 July 2015 Published online: 24 July 2015

\section{References}

1. Hartley PG, Galvin JR, Hunninghake GW, Merchant JA, Yagla SJ, Speakman $\mathrm{SB}$, et al. High-resolution CT-derived measures of lung density are valid indexes of interstitial lung disease. J Appl Physiol. 1994;76:271-7.

2. Best AC, Lynch AM, Bozic CM, Miller D, Grunwald GK, Lynch DA. Quantitative $C T$ indexes in idiopathic pulmonary fibrosis: relationship with physiologic impairment. Radiology. 2003;228:407-14.

3. Do KH, Lee JS, Colby TV, Kitaichi M, Kim DS. Nonspecific interstitial pneumonia versus usual interstitial pneumonia: differences in the density histogram of high-resolution CT. J Comput Assist Tomogr. 2005;29:544-8.

4. Camiciottoli G, Orlandi I, Bartolucci M, Meoni E, Nacci F, Diciotti S, et al. Lung CT densitometry in systemic sclerosis: correlation with lung function, exercise testing, and quality of life. Chest. 2007;131:672-81.

5. Best AC, Meng J, Lynch AM, Bozic CM, Miller D, Grunwald GK, et al. Idiopathic pulmonary fibrosis: physiologic tests, quantitative $C T$ indexes, and CT visual scores as predictors of mortality. Radiology. 2008;246:935-40.

6. Handa T, Nagai S, Hirai T, Chin K, Kubo T, Oga T, et al. Computed tomography analysis of airway dimensions and lung density in patients with sarcoidosis. Respiration. 2009;77:273-81.

7. Raghu G, Collard HR, Egan JJ, Martinez FJ, Behr J, Brown KK, et al. An official ATS/ERS/JRS/ALAT statement: idiopathic pulmonary fibrosis: evidence-based guidelines for diagnosis and management. Am J Respir Crit Care Med. 2011;183:788-824.

8. Lynch DA, Travis WD, Muller NL, Galvin JR, Hansell DM, Grenier PA, et al. Idiopathic interstitial pneumonias: CT features. Radiology. 2005;236:10-21.

9. American Thoracic Society; European Respiratory Society. American Thoracic Society/European Respiratory Society International Multidisciplinary Consensus Classification of the Idiopathic Interstitial Pneumonias. This joint statement of the American Thoracic Society (ATS), and the European Respiratory Society (ERS) was adopted by the ATS board of directors, June 2001 and by the ERS Executive Committee, June 2001. Am J Respir Crit Care Med. 2002;165:277-304.

10. Travis WD, Costabel U, Hansell DM, King Jr TE, Lynch DA, Nicholson AG, et al. An official American Thoracic Society/European Respiratory Society statement: Update of the international multidisciplinary classification of the idiopathic interstitial pneumonias. Am J Respir Crit Care Med. 2013;188:733-48.

11. Miller MR, Hankinson J, Brusasco V, Burgos F, Casaburi R, Coates A, et al. Standardisation of spirometry. Eur Respir J. 2005;26:319-38.
12. The Committee of Pulmonary Physiology JRS. Guidelines for Pulmonary Function Tests: Spirometry, Flow-Volume Curve, Diffusion Capacity of the Lung. Tokyo: The Japanese Respiratory Society; 2004.

13. Ohara T, Hirai T, Sato S, Terada K, Kinose D, Haruna A, et al. Longitudinal study of airway dimensions in chronic obstructive pulmonary disease using computed tomography. Respirology. 2008;13:372-8.

14. Hansell DM, Bankier AA, MacMahon H, McLoud TC, Muller NL, Remy J. Fleischner Society: glossary of terms for thoracic imaging. Radiology. 2008;246:697-722.

15. Kazerooni EA, Martinez FJ, Flint A, Jamadar DA, Gross BH, Spizarny DL, et al. Thin-section CT obtained at 10-mm increments versus limited three-level thin-section CT for idiopathic pulmonary fibrosis: correlation with pathologic scoring. AJR Am J Roentgenol. 1997;169:977-83.

16. Lynch DA, Godwin JD, Safrin S, Starko KM, Hormel P, Brown KK, et al. High-resolution computed tomography in idiopathic pulmonary fibrosis: diagnosis and prognosis. Am J Respir Crit Care Med. 2005;172:488-93.

17. Iwasawa T, Asakura A, Sakai F, Kanauchi T, Gotoh T, Ogura T, et al. Assessment of prognosis of patients with idiopathic pulmonary fibrosis by computer-aided analysis of CT images. J Thorac Imaging. 2009;24:216-22.

18. Sumikawa H, Johkoh T, Yamamoto S, Yanagawa M, Inoue A, Honda O, et al. Computed tomography values calculation and volume histogram analysis for various computed tomographic patterns of diffuse lung diseases. J Comput Assist Tomogr. 2009;33:731-8.

19. Sumikawa H, Johkoh T, Colby TV, Ichikado K, Suga M, Taniguchi H, et al. Computed tomography findings in pathological usual interstitial pneumonia: relationship to survival. Am J Respir Crit Care Med. 2008;177:433-9.

20. Sumikawa H, Johkoh T, Yamamoto S, Oota M, Ueguchi T, Ogata Y, et al. Volume histogram analysis for lung thin-section computed tomography: differentiation between usual interstitial pneumonia and nonspecific interstitial pneumonia. J Comput Assist Tomogr. 2007;31:936-42.

21. Tanabe N, Muro S, Hirai T, Oguma T, Terada K, Marumo S, et al. Impact of exacerbations on emphysema progression in chronic obstructive pulmonary disease. Am J Respir Crit Care Med. 2011;183:1653-9.

22. Haruna A, Muro S, Nakano Y, Ohara T, Hoshino Y, Ogawa E, et al. CT scan findings of emphysema predict mortality in COPD. Chest. 2010;138:635-40.

23. Ohara T, Hirai T, Muro S, Haruna A, Terada K, Kinose D, et al. Relationship between pulmonary emphysema and osteoporosis assessed by $\mathrm{CT}$ in patients with COPD. Chest. 2008;134:1244-9.

24. Matsumoto H, Niimi A, Takemura M, Ueda T, Minakuchi M, Tabuena R, et al. Relationship of airway wall thickening to an imbalance between matrix metalloproteinase-9 and its inhibitor in asthma. Thorax. 2005;60:277-81.

25. Nakano Y, Muro S, Sakai H, Hirai T, Chin K, Tsukino M, et al. Computed tomographic measurements of airway dimensions and emphysema in smokers. Correlation with lung function. Am J Respir Crit Care Med. 2000;162:1102-8.

26. Mishima M, Hirai T, Itoh H, Nakano Y, Sakai H, Muro S, et al. Complexity of terminal airspace geometry assessed by lung computed tomography in normal subjects and patients with chronic obstructive pulmonary disease. Proc Natl Acad Sci U S A. 1999;96:8829-34.

27. Maldonado F, Moua T, Rajagopalan S, Karwoski RA, Raghunath S, Decker PA, et al. Automated quantification of radiological patterns predicts survival in idiopathic pulmonary fibrosis. Eur Respir J. 2014;43:204-12.

28. Watadani T, Sakai F, Johkoh T, Noma S, Akira M, Fujimoto K, et al. Interobserver variability in the $C T$ assessment of honeycombing in the lungs. Radiology. 2013;266:936-44.

29. Sumikawa H, Johkoh T, Nagareda T, Sekiguchi J, Matsuo K, Fujita Y, et al. Pulmonary adenocarcinomas with ground-glass attenuation on thin-section $\mathrm{CT}$ : quantification by three-dimensional image analyzing method. Eur J Radiol. 2008:65:104-11.

30. Martinez FJ, de Andrade JA, Anstrom KJ, King Jr TE, Raghu G, Idiopathic Pulmonary Fibrosis Clinical Research N. Randomized trial of acetylcysteine in idiopathic pulmonary fibrosis. N Engl J Med. 2014;370:2093-101.

31. Nishiyama O, Taniguchi $\mathrm{H}$, Kondoh Y, Kimura T, Kato K, Kataoka K, et al. A simple assessment of dyspnoea as a prognostic indicator in idiopathic pulmonary fibrosis. Eur Respir J. 2010:36:1067-72.

32. Lama VN, Flaherty KR, Toews GB, Colby TV, Travis WD, Long Q, et al. Prognostic value of desaturation during a 6-minute walk test in idiopathic interstitial pneumonia. Am J Respir Crit Care Med. 2003;168:1084-90.

33. Ley B, Collard HR, King Jr TE. Clinical course and prediction of survival in idiopathic pulmonary fibrosis. Am J Respir Crit Care Med. 2011;183:431-40. 
34. du Bois RM, Weycker D, Albera C, Bradford WZ, Costabel U, Kartashov A, et al. Ascertainment of individual risk of mortality for patients with idiopathic pulmonary fibrosis. Am J Respir Crit Care Med. 2011;184:459-66.

35. Hamada K, Nagai S, Tanaka S, Handa T, Shigematsu M, Nagao T, et al. Significance of pulmonary arterial pressure and diffusion capacity of the lung as prognosticator in patients with idiopathic pulmonary fibrosis. Chest. 2007;131:650-6.

36. Collard HR, King Jr TE, Bartelson BB, Vourlekis JS, Schwarz MI, Brown KK. Changes in clinical and physiologic variables predict survival in idiopathic pulmonary fibrosis. Am J Respir Crit Care Med. 2003;168:538-42.

37. Lee JS, Ryu JH, Elicker BM, Lydell CP, Jones KD, Wolters PJ, et al. Gastroesophageal reflux therapy is associated with longer survival in patients with idiopathic pulmonary fibrosis. Am J Respir Crit Care Med. 2011;184:1390-4.

38. Jegal Y, Kim DS, Shim TS, Lim CM, Do Lee S, Koh Y, et al. Physiology is a stronger predictor of survival than pathology in fibrotic interstitial pneumonia. Am J Respir Crit Care Med. 2005;171:639-44.

39. Taniguchi H, Kondoh Y, Ebina M, Azuma A, Ogura T, Taguchi Y, et al. The clinical significance of $5 \%$ change in vital capacity in patients with idiopathic pulmonary fibrosis: extended analysis of the pirfenidone trial. Respir Res. 2011;12:93.

\section{Submit your next manuscript to BioMed Central and take full advantage of:}

- Convenient online submission

- Thorough peer review

- No space constraints or color figure charges

- Immediate publication on acceptance

- Inclusion in PubMed, CAS, Scopus and Google Scholar

- Research which is freely available for redistribution 